\title{
Evolution of Reovirus in Cancer Therapy
}

\author{
*Bindhu Jayaprakash, **Sivapriya Veeraiyan, ***Silambarasan Gunaseelan, \\ ****Yasodha Purushothaman \\ *Research Associate cum Assistant Professor \\ * *Research Associate cum Assistant Professor \\ ***M.Tech Student \\ ****M.Tech Student, Dept. of Biotechnology, Bannari Amman Institute of Technology, Sathy-638401
}

\begin{abstract}
The overactive Ras signaling can ultimately lead to cancer. The 3 Ras genes in humans (HRas, KRas, and NRas) are the most common oncogenes in human cancer.Mutations that permanently activate Ras are found in $20 \%$ to $25 \%$ of all human tumors and up to $90 \%$ in certain types of cancer (e.g., pancreatic cancer). For this reason, Ras inhibitors are being studied as a treatment for cancer and other diseases with Ras overexpression. Reovirus was noted to be a potential cancer therapeutic when studies suggested it reproduces well in certain cancer cell lines. It replicates specifically in cells that have an activated Ras pathway (a cellular signaling pathway that is involved in cell growth and differentiation).Reovirus replicates in and eventually kills Ras-activated tumour cells and as cell death occurs, progeny virus particles are free to infect surrounding cancer cells. This cycle of infection, replication and cell death is believed to be repeated until all tumour cells carrying an activated Ras pathway are destroyed.Reovirus, a double-stranded ribonucleic acid virus and benign human pathogen, preferentially infects and kills cancer cells in its unmodified form, and is one of the leading oncolytic viruses currently undergoing clinical trials internationally. Importantly, reovirus has been shown to be effective as a monotherapy, as well as in combination with other anticancer options, including radiation and chemotherapeutic agents, such as gemcitabine, docetaxel, paclitaxel, and carboplatin.
\end{abstract}

Keywords: Reovirus, monotherapy, anticancer

\section{Introduction}

With 32 clinical trials completed or ongoing thus far, reovirus has demonstrated clinical therapeutic applicability against a multitude of cancers, including but not limited to breast cancer, prostate cancer, pancreatic cancer, malignant gliomas, advanced head and neck cancers, and metastatic ovarian cancers. ${ }^{[1]}$ Phase I trials have demonstrated that reovirus is safe to use via both intralesional/intratumoral and systemic routes of administration, with the most common adverse reactions being grade I/II toxicities, such as flu-like illness (fatigue, nausea, vomiting, headache, fever/chills, dizziness), diarrhea, and lymphopenia. In subsequent Phase II trials, reovirus administration was demonstrated to successfully decrease tumor size and promote tumor necrosis, thereby complementing compelling preclinical evidence of tumor destruction by the virus. Of note, the first Phase III clinical trial using reovirus in combination with paclitaxel and carboplatin for the treatment of head and neck cancers is under way. ${ }^{[2,3]}$ Based on the evidence from clinical trials, we comprehensively review the use of reovirus as an anticancer agent, acknowledge key obstacles, and suggest future directions to ultimately potentiate the efficacy of reovirus oncotherapy.Oncolytic viruses (OVs) preferentially target and kill cancer cells through a process known as oncolysis, while leaving normal, untransformed cells relatively unaffected. The susceptibility of a particular cell to OV infection and subsequent oncolysis stems from the aberrant cellular signaling and defective immune responses, which are inherently common in cancer cells. Currently, numerous viruses, in their natural or modified form, have been shown to possess oncolytic properties, and thus are being studied for their potential use as anticancer agents. Some prominent examples of these OVs include reovirus ${ }^{[4]}$ Newcastle disease,${ }^{[5]}$ vesicular stomatitis ${ }^{[6]}$ vaccinia,${ }^{[7]}$ measles,${ }^{[8]}$ poliovirus, ${ }^{[9]}$ herpessimplex,${ }^{[10]}$ adenovirus, ${ }^{[11]}$ Maraba,${ }^{[12][13]}$ and Coxsackie.${ }^{[14}$ Although the use of reovirus in cancer therapy stems from the oncolytic capabilities of the virus, recent studies have illustrated that reovirus additionally invokes a sequence of immunological events that ultimately overturn various tumor-induced immunosuppressive mechanisms and promotes the development of an antitumor immune response. ${ }^{[34-39]}$ Studies have shown that reovirus treatment promotes the secretion of a range of proinflammatory cytokines and chemokines following administration. ${ }^{[34,38,40]}$ Additionally, upon exposure to reovirus, dendritic cells (DCs) produce various proinflammatory cytokines, undergo maturation, and migrate into the tumor microenvironment along with $\mathrm{CD} 8^{+} \mathrm{T}$ cells. ${ }^{[34,39]}$ These reovirus-activated DCs possess the ability to prime tumor antigen-specific $\mathrm{T}$ cells (both in vitro and in vivo) ${ }^{[34]}$ and increase the cytolytic activity of innate immune cells (natural killer $[\mathrm{NK}]$ cells) ${ }^{[39]}$ Such a robust reovirus-mediated antitumor immunity can also protect the host against subsequent tumor challenge, even after discontinuation of therapy. ${ }^{[34]}$ Collective evidence thus far suggests that reovirus- 
based cancer therapy targets cancer by direct oncolysis and antitumor immune activities, both of which are essential to achieve optimal tumor regression and clinical outcomes. In this context, this review focuses on preclinical and clinical studies with reovirus as a single agent or in combination therapy for treatment of cancers. We highlight key findings from the studies on reovirus oncotherapy and identify major obstacles that if appropriately managed would dictate the successful translation of reovirus oncotherapy into clinical practice.

\section{Preclinical studies with Reovirus:}

Duncan et al noted that simian virus (SV-40)-transformed human embryonic lung cells (WI-38 cells) had increased sensitivity to reovirus cytotoxicity in comparison to untransformed WI-38 cells. ${ }^{[42]}$ Besides Hashiro et al also observed early connections with reovirus and its oncolytic properties, and found that certain tumor cells and spontaneously transformed cell lines (human and murine) have preferential susceptibility toward the cytotoxic effects of reovirus. ${ }^{[41]}$ Although these studies revealed the oncolytic properties of reovirus, it was not until two decades later when Coffey et al published a paper in Science that the possibility of using reovirus as an anticancer agent was entertained. ${ }^{\underline{1}}$ This article revealed that a single intratumoral injection of reovirus could result in the regression of established v-erbB-transformed NIH 3T3 or human U87 glioblastoma tumors in $80 \%$ of severe combined immunodeficient (SCID) mice. ${ }^{1}$ Furthermore, Coffey et al using an immunocompetent mouse model (Ras-transformed C3H-10T1/2 fibroblasts implanted into the flank of $\mathrm{C} 3 \mathrm{H}$ mice), they also illustrated that a therapeutic regimen of multiple reovirus injections resulted in complete regression in $65 \%$ of the tumors. ${ }^{[4]}$

Given the frequency of mutations in Ras and associated pathways in human cancers, as well as the relatively nonpathogenic nature of reovirus, it was evident that reovirus was an attractive anticancer agent. Following the discovery of the oncolytic potential of reovirus, subsequent studies focused on expanding the applicability of reovirus as a single agent against multiple types of cancers in various models. A study by Hata et al found that reovirus could kill six human breast cancer cell lines (SK-BR-3, KPL4, MDA-MB-453, CRL1500, MCFT, and MDA-MB-231) but not a normal breast cell line (Hs578Bst). ${ }^{[15]}$ Complementing this study, it was also illustrated that reovirus could effectively shrink MDA-MB-453 tumors in SCID mice, including tumors situated remotely from the reovirus injection site. ${ }^{[16]}$ In addition to breast cancer, reovirus oncotherapy is effective in brain, lymphoma, ovarian, spinal, bladder, and colon-derived cancer cells and cancer stem cells..$^{[17,20,24,26,27,43]}$ One particular study treated low-passage cell lines derived from nine surgically excised human gliomas, and demonstrated that ex vivo reovirus treatment killed $100 \%$ of specimens. ${ }^{[20]}$ Although the majority of these preclinical studies were conducted in vitro or in SCID/nude mouse models in vivo, it was also illustrated that reovirus had beneficial anticancer effects in immunocompetent mouse models ${ }^{[4,44]}$ especially in the presence of immunosuppressant agents, such as cyclosporine A or anti-CD4/anti-CD8 antibodies. ${ }^{[44]}$ Therefore, reovirus oncotherapy has the potential to be utilized for the treatment of a wide range of cancer types and may benefit more with a combination approach, especially in immunocompetent hosts. Early studies using reovirus as a single anticancer agent showed impressive preclinical therapeutic efficacy. However, the efficacy of reovirus monotherapy was suboptimal in immunocompetent hosts. To address this issue, reovirus was combined with various anticancer treatment options, such as chemotherapeutic agents or radiotherapy, to achieve enhanced efficacy. Interestingly, these combination studies revealed that the use of reovirus along with common chemotherapeutic agents, such as cisplatin, docetaxel, or cisplatin-paclitaxel doublet chemotherapy or radiotherapy, had synergistic effects in vitro and in vivo. ${ }^{[21-23], 45]}$ For example, a study conducted by Sei et al investigated the in vitro combination effects of reovirus with a variety of chemotherapeutic agents (cisplatin, gemcitabine, vinblastine, and paclitaxel) against human non-small-cell lung cancer (NSCLC) cells. ${ }^{[25]}$ Additionally, strong synergistic effects were observed only in those cell lines that were sensitive to monotherapy of the respective chemotherapeutic agent. ${ }^{[25]}$ In contrast, treatment of NSCLC cell lines with the reovirus and paclitaxel combination treatment was synergistic in all cell lines regardless of their sensitivity to either agent alone ${ }^{[25]}$ This enhanced treatment efficacy was suggested to be the result of accelerated apoptosis triggered by paclitaxel-mediated mitotic arrest. ${ }^{[25]}$ This suggests that certain changes in programmed cell-death pathways may be advantageous in enhancing the efficacy of reovirus therapy. In agreement with this hypothesis, recent studies have also shown that activation or stabilization of programmed cell-death pathways further enhances reovirus-induced apoptosis. ${ }^{[18,46]}$ In particular, actinomycin D, etoposide, or nutlin-3a have all shown enhanced reovirus-induced apoptosis via p53-dependent NF- $\mathrm{BB}$ activation ${ }^{[18,46]}$ Therefore, if properly chosen, a combination therapeutic approach, with lower chemotherapeutic dosages compared to monotherapy trials, could enhance reovirus-mediated cytotoxicity while reducing drug toxicity.

\section{Clinical studies with Reovirus:}

There have been a total of 32 clinical trials - 15 completed and 17 ongoing - internationally in countries including Canada, the US, and the UK. Of all the clinical trials, six are in partnership with the US 
National Cancer Institute, and four are in partnership with the National Cancer Institute of Canada Clinical Trials Group. So far, 308 patients have been treated with reovirus in completed studies; approximately 610 patients are currently enrolled in ongoing trials, and it is expected that an additional 631 patients will be enrolled in future trials. Reovirus has been or will be used in 12 clinical trials as a monotherapy and in 20 clinical trials in combination with other anticancer agents. Since 2000, clinical-grade formulation of the type 3 Dearing strain of wild-type reovirus (Reolysin ${ }^{\circledR}$; Oncolytics Biotech, Calgary, Canada ${ }^{44}$ ) has been tested in various clinical trials for the treatment of a multitude of cancers, such as prostate cancer, ${ }^{[48-50]}$ malignant gliomas, ${ }^{[51-53]}$ advanced head and neck cancers, ${ }^{[54-57]}$ and metastatic ovarian cancers, ${ }^{[58-60]}$ among others

\section{Monotherapy:}

The first Phase I clinical trial to use reovirus was an intralesional monotherapy for the treatment of 18 patients with advanced solid tumors, including soft-tissue sarcomas, melanoma, breast cancer, and head and neck cancers. ${ }^{[61]}$ This study was designed to determine the safety and tolerability of reovirus percutaneous intralesional administration in patients with prior therapeutic interventions. Patients were monitored for 6 weeks, toxicities were measured according to the National Cancer Institute Clinical Trials Group expanded common toxicity criteria, and tumor responses were measured using the Response Evaluation Criteria in Solid Tumors (RECIST). After 6 weeks, one patient showed a complete response (CR) and two demonstrated partial responses (PRs), while four had stable disease (SD) and ten showed progressive disease (PD). The results showed for the first time that reovirus administered intralesionally up to $1 \times 10^{10}$ plaque-forming units as a monotherapy is safe and well tolerated without reaching dose-limiting toxicities (DLTs) or maximum tolerated dosages (the dose at which two or more subjects out of six in a dose group experience DLT). Based on the safety data from this first trial, a translational study was designed to assess the oncolytic activity (ie, the efficacy) of reovirus in preclinical models and in six prostate cancer patients. ${ }^{[48]}$ In this study, the prostate cancer patients first received a single reovirus injection of $1 \times 10^{7}$ plaque-forming units, and 3 weeks later underwent prostatectomies. Importantly, the immunohistochemical analysis of resected prostate tissues from these patients revealed a preference of reovirus to infect cancerous tissue rather than noncancerous tissue. The same analysis also demonstrated apoptosis and lymphocyte infiltration in reovirus-treated cancerous tissues. Together, the results from this trial supported the preclinical findings that reovirus preferentially targets and kills cancer cells and also promotes immunomodulation in the cancer milieu. Two subsequent combination Phase I/II studies were performed using local administration of reovirus for recurrent malignant gliomas, and confirmed that intratumoral administration of reovirus is well tolerated as a monotherapy. ${ }^{[51,52]}$

Next, two clinical trials investigated whether reovirus could be safely administered systemically to treat advanced malignancies and their associated metastases. In the first study, of the 16 of 18 patients that were evaluable for the objective response by imaging studies as determined by RECIST, one patient showed a PR with a 34\% shrinkage in tumor burden, and seven patients demonstrated SD irrespective of dose level. ${ }^{[62]}$ Histological examination of the posttreatment biopsy samples showed extensive necrosis that was accompanied by markers of viral replication and remnant viral particles. Similarly, the second study treated 33 patients, three of whom had reduced levels of tumor markers (carcinoembryonic antigen, prostate-specific antigen, and carbohydrate antigen 19.9) following reovirusadministration. ${ }^{[63]}$ Computed tomography also revealed evidence of tumor necrosis in one patient, quantified as a $12 \%$ reduction, and biopsy analysis confirmed viable virus in three patients where recovered reovirustiter directly correlated with the dosage administered. Taken together, the results of these two Phase I trials using systemically administered reovirus supported the previously reported antitumor activities of reovirus, and led to the conclusion that monthly intravenous injections of reovirus are safe and well tolerated in patients with advanced malignancies.

The first Phase II clinical trial for intravenous reovirus monotherapy began enrollment in 2008 for the treatment of metastatic melanoma. ${ }^{[64]}$ Twenty-one patients were injected intravenously with reovirus at a dose of $3 \times 10^{10} 50 \%$ tissue-culture infective dose $\left(\mathrm{TCID}_{50}\right)$ once every 60 minutes on days $1-5$ every 4 weeks, and were monitored for clinical benefits of CR or PR for at least 8 weeks. Although no patients met the criteria for CR or PR, one patient did demonstrate extensive (75\%-90\%) tumor necrosis in two metastatic lesions following two treatment cycles. Additionally, productive reovirus replication was detected in two of 13 biopsies that contained melanoma metastases and were performed 1 week following treatment, further supporting the positive clinical outcomes from the earlier Phase I results. ${ }^{[62,67]}$ Additional evidence was gathered for the safety of reovirus as there were few severe (grade III or IV) toxicities reported. Furthermore, two of 13 patients demonstrated productive reoviral replication in melanoma metastases; however, the study's clinical objective to achieve at least two or more patients having a CR or PR was not met, and thus the trial could not proceed as planned. Therefore, this Phase II trial did not support the use of reovirus as a monotherapy for metastatic melanoma, but the evidence gathered suggested that reovirus may be more efficacious as part of a combination therapy with other chemotherapeutic agents. In contrast, another Phase II clinical trial for intravenous administration of reovirus for 52 patients with lung metastases of soft-tissue sarcomas demonstrated a total clinical benefit rate of 
$43 \%$ (ie, 19 of 44 evaluable patients experienced stable disease for as short as 2 months but as long as 22 months). ${ }^{[65]}$ Positive results from this completed trial showed promise for intravenous reovirus therapy for metastatic sarcomas; however, like the aforementioned Phase II trial, they suggested that a combination of reovirus with other therapeutic options could greatly enhance reovirus therapy and promote better patient outcomes.

There are three ongoing clinical trials for reovirus as a monotherapy. The first began in 2008, and is a Phase I/II combined clinical trial conducted in partnership with the US National Cancer Institute for intravenous and intraperitoneal administration of reovirus for patients with metastatic ovarian, peritoneal, and fallopian tube cancers that have not responded to platinum chemotherapy. ${ }^{[58]}$ Thus far, the study has concluded that there is evidence of selective reovirus penetration in peritoneal tumors, and no DLTs had been observed as of 2010. The second ongoing trial, performed by the University of Leeds in the UK, is a Phase I trial in which the intravenous administration of reovirus is done prior to surgery in patients with recurrent high-grade primary or metastatic brain tumors. ${ }^{[66]}$ The third trial is a Phase I clinical trial for intravenous administration of reovirus for patients with relapsed multiple myeloma, and has an estimated primary completion date of December $2014 .{ }^{[67,68]}$ This is the first clinical trial to examine the use of reovirus as a treatment for hematological malignancies.

Thus far, the most frequently reported toxicities from reovirus have been low-grade (I or II) flu-like symptoms. Adverse events due to reovirus include nausea, vomiting, headache, fever/chills, diarrhea, anorexia, and weight loss; however, all appear to be independent of treatment dose or cycle. Two studies using intratumoral administration of reovirus showed no severe toxicities at all associated with reovirus. ${ }^{[48,51]}$ Most toxicities, when observed, occur early in the treatment regimen and were better tolerated in the later cycles. Some studies show hematological abnormalities, including lymphopenia and neutropenia often of grade III or IV; however, these occurrences were few and were usually unlikely to be related to reovirus, and typically did not result in negative clinical consequences. ${ }^{[61,63,69]}$ In contrast, other studies showed no evidence of hematological toxicities at all. ${ }^{[62,70]}$ Taken together, toxicity results indicate that reovirus is well tolerated by patients, with generally only mild manageable adverse events.

\section{Combination therapy with reovirus in clinical studies}

Both preclinical and clinical studies thus far have demonstrated a higher efficacy for reovirus cancer therapy when reovirus is combined with other forms of cancer therapy, including radiotherapy and single- and multiple-chemotherapy regimens. Not surprisingly, the majority of ongoing clinical trials involve the administration of reovirus in combination with an additional anticancer therapeutic agent.

The first combination reovirus clinical trial treated patients with advanced cancers, including one of unknown primary origin, melanoma, head and neck cancer, squamous cell carcinoma of the skin, lung, ovarian, colorectal, esophageal, and pancreatic cancer in a two-stage Phase I dose-escalation study using intratumoralreovirus and palliative radiotherapy. ${ }^{[71]}$ Here, reovirus doses ranging from $1 \times 10^{8}$ to $1 \times 10^{10} \mathrm{TCID}_{50}$ and radiation levels of 20-36 Gy were used in combinations. This study concluded that reovirus antitumor activity is unaffected by high doses of radiation, and that the combination of the two is safe for further investigations. Following the initial study, a subsequent Phase II trial ${ }^{69}$ was performed using low-dose radiation, and demonstrated a 93\% total disease-control rate (combined CR, PR, and SD) in treated lesions, thereby confirming the safety and positive clinical outcomes of the combination regimen. As opposed to palliative radiotherapy, it is expected that reovirus will be used in future trials in combination with radical radiotherapy for a curative intent.

The first chemotherapeutic agent used in combination with reovirus was gemcitabine for the treatment of 16 patients with advanced malignancies, including squamous cell, undifferentiated, and poorly differentiated carcinoma, cholangiocarcinoma, esophageal adenocarcinoma, fibrosarcoma, NSCLC, and colorectal, breast, and cervical cancer. ${ }^{[73]}$ Through this study, the concluding recommended dose for this particular combination was as follows: reovirus $1 \times 10^{10} \mathrm{TCID}_{50}$ on day 1 and gemcitabine at $1,000 \mathrm{mg} / \mathrm{m}^{2}$ on day 1 and day 8 of a 21 -day cycle. Of the ten evaluable patients, one had a PR and a minor decrease in tumor size after just four cycles of therapy. Although this combination was determined to be safe enough for further studies, this trial highlighted the importance of potential safety issues when combining reovirus with immunomodulatory agents that enhance antitumor immune responses. This study cautions that a fine balance must be achieved between agents that dampen antiviral immune responses, as they could potentially exacerbate reovirus toxicities and pose a minor but real threat to patient safety. For instance, the ongoing Phase I clinical trial using cyclophosphamide, a known immunosuppressant drug, in combination with reovirus (REO 012) includes a primary objective of determining the minimum effective immunomodulatory dose of cyclophosphamide. ${ }^{[74]}$ Here, it will be important to monitor the effect of cyclophosphamide on antiviral immune responses and the potential for exacerbation of reovirus toxicities. A Phase II clinical trial is ongoing to further evaluate the interactions between reovirus and gemcitabine in a clinical setting. ${ }^{[75-77]}$ 
Other chemotherapeutic agents that have completed at least Phase I clinical trials in combination with reovirus include docetaxel, ${ }^{[78]}$ and paclitaxel and carboplatin. ${ }^{[69]}$ All aforementioned combinations have gone on to further Phase II or Phase III clinical trials. In either monotherapy or combination therapy studies, the recommended dose of reovirus is $3 \times 10^{10} \mathrm{TCID}_{50}$ once over 60 minutes on days $1-5$ every 3 or 4 weeks. The recommended dose of docetaxel in combination therapy is $75 \mathrm{mg} / \mathrm{m}^{2}$ three times weekly. ${ }^{[49,50,78]}$ As for paclitaxel and carboplatin, paclitaxel is recommended at a dose of $175 \mathrm{mg} / \mathrm{m}^{2}$, and carboplatin at an area under the curve (AUC) of $5^{[54,55,69,79]}$ or paclitaxel at $200 \mathrm{mg} / \mathrm{m}^{2}$ and carboplatin at AUC $6^{[79,80]}$ on day 1 of a 3-week cycle. Other chemotherapeutic agents currently in or recruiting patients for Phase I trials with reovirus include cyclophosphamide, ${ }^{[74,81,82]}$ FOLFIRI (folinic acid, fluorouracil, irinotecan)/bevacizumab, ${ }^{[83,84]}$ paclitaxel alone $^{[85,86]}$, FOLFOX (folinic acid, fluorouracil, oxaliplatin)-6/bevacizumab, ${ }^{84,85}$ docetaxel and prednisone, ${ }^{[49,50]}$ and docetaxel and pemetrexed. ${ }^{[89,90]}$

Overall, combination therapies have demonstrated that reovirus does not exacerbate known chemotherapy or radiation-associated toxicities or vice versa. ${ }^{[69,71,78]}$ However, the known toxicities of gemcitabine and reovirus were observed in one trial. ${ }^{[73]}$ Lolkema et al concluded that since gemcitabine appears to have an inhibitory effect on the development of neutralizing antireoviral antibodies (NARAs), a chemotherapeutic regimen in combination with reovirusmay exacerbate reovirus toxicity in the liver and further elevate liver enzyme levels. ${ }^{[73]}$

\section{Key obstacles to reovirus oncotherapy}

With a total of 32 clinical trials (completed or ongoing) and continued progress in preclinical studies, reovirus therapy is well set to transition from "bench" to "bedside" for the treatment of a multitude of cancers. At the same time, these studies have also recognized key obstacles that will in future dictate the successful translation of reovirus oncotherapy into clinical practice. These key obstacles are associated with antiviral immune responses and delivery of reovirus to cancer cells.

Host immune responses pose the biggest challenge for reovirus oncotherapy. Reovirus-induced immunological events in tumor-bearing hosts are very complex and unique, as they involve the opposing and concurrent effects of antiviral and antitumor immunity. Type I IFNs, produced immediately following the exposure of host to reovirus, can directly inhibit virus replication. ${ }^{[30,91]}$ Furthermore, such IFNs in combination with other proinflammatory cytokines (ie, TNF $\alpha$, IL-1, IL-6, IL-12, etc) augment the innate immune response through the recruitment and activation of immune cells, such as neutrophils, macrophages, DCs, and NK cells, ${ }^{[92]}$ and hamper reoviral spread. Similarly, antiviral adaptive immunity, wherein $\mathrm{T}$ cells contain viral replication via direct killing of virally infected cells or by the production of cytokines, such as IFN $\gamma^{90}$ and $\mathrm{B}$ cells, produce NARAs and curtail the replication and spread of reovirus prematurely before it can eliminate all the cancer cells. It should be noted that NARAs are prevalent in most adults, owing to exposure to reovirus sometime during their lifetime ${ }^{[48,62-64]}$ Therefore, future directions need to focus on specifically targeting the antiviral immune response, such as $\mathrm{T}$-cell and $\mathrm{B}$ cell responses, without affecting the production of strong antitumor immunity.

The other related challenge centers on the delivery of reovirus to tumor cells. Although intratumoral injections are designed to deliver all of the virus particles to the tumor vicinity, systemic viral administration has several advantages. In particular, systemic delivery increases the probability that the virus can reach metastatic tumors or tumors that constitute multiple nodules that are not confined to a particular area. Unfortunately, various host factors, programmed to restrict the spread of pathogens, such as NARAs, nonspecific uptake by organs (spleen, lung, and liver) and scavenging immune cells, and poor virus escape from the vascular compartment, ${ }^{[96,97]}$ significantly affect virus delivery through systemic routes. As reviewed by Roy et al, multiple steps have been attempted to ameliorate the systemic delivery of OVs with the use of cell carriers. ${ }^{[98]}$ It is noteworthy that immune cells loaded with reovirus can shield the virus from circulating NARAs and deliver it to tumor cells, ${ }^{[95,99]}$ and represent a clinically feasible means of enhancing systemic reovirus delivery to the tumor cells.

\section{Conclusion}

Reovirus oncotherapy has shown definitive promise through preclinical and clinical trials, and is en route to becoming a clinically practiced therapeutic option for the treatment of various cancers. In this review, we hope to have clearly outlined the progress that has been made in reovirus clinical trials, from monotherapies to combination trials, by providing up-to-date trial results from completed, ongoing, and upcoming studies. In future, a major thrust of preclinical research should be on overcoming the current obstacles to reovirus oncotherapy, and translating that knowledge to clinical trials.

Considering the focus of this article, we have not addressed the immunological aspects of. On one hand, antiviral immune responses hamper the efficacy of oncotherapy. On the other hand, antitumor immunity further potentiates anticancer effects. It is clear that research into oncotherapy-driven immune responses is a 
major priority, as the strategic management of antiviral and antitumor immune responses holds the key to achieving optimum efficacy of reovirus oncotherapy.

\section{References}

[1] Goodsell DS (1999). "The molecular perspective: the ras oncogene". Oncologist. 4 (3): 263-4. PMID 10394594.

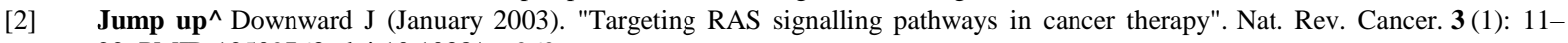
22. PMID 12509763. doi:10.1038/nrc969.

[3] 24 Lal R, Harris D, Postel-Vinay S, de Bono J (October 2009). "Reovirus: Rationale and clinical trial update". Curr. Opin. Mol. Ther. 11 (5): 532-9. PMID 19806501.

[4] Coffey MC, Strong JE, Forsyth PA, Lee PW. Reovirus therapy of tumors with activated Ras pathway. Science. 1998;282(5392):1332-1334. [PubMed]

[5] Schirrmacher V, Fournier P. Newcastle disease virus: a promising vector for viral therapy, immune therapy, and gene therapy of cancer. Methods Mol Biol. 2009;542:565-605. [PubMed]

[6] Barber GN. VSV-tumor selective replication and protein translation. Oncogene. 2005;24(52):7710-7719. [PubMed]

[7] Kirn DH, Thorne SH. Targeted and armed oncolytic poxviruses: a novel multi-mechanistic therapeutic class for cancer. Nat Rev Cancer. 2009;9(1):64-71. [PubMed]

[8] Msaouel P, Iankov ID, Dispenzieri A, Galanis E. Attenuated oncolytic measles virus strains as cancer therapeutics. Curr Pharm Biotechnol. 2012;13(9):1732-1741. [PMC free article] [PubMed]

[9] Goetz C, Gromeier M. Preparing an oncolytic poliovirus recombinant for clinical application against glioblastoma multiforme. Cytokine Growth Factor Rev. 2010;21(2-3):197-203. [PMC free article][PubMed]

[10] Kaur B, Chiocca EA, Cripe TP. Oncolytic HSV-1 virotherapy: clinical experience and opportunities for progress. Curr Pharm Biotechnol. 2012;13(9):1842-1851. [PMC free article] [PubMed]

[11] Ko D, Hawkins L, Yu DC. Development of transcriptionally regulated oncolytic adenoviruses. Oncogene. 2005;24(52):77637774. [PubMed]

[12] Brun J, McManus D, Lefebvre C, et al. Identification of genetically modified maraba virus as an oncolytic rhabdovirus. MolTher. 2010;18(8):1440-1449. [PMC free article] [PubMed]

[13] Pol JG, Zhang L, Bridle BW, et al. Maraba virus as a potent oncolytic vaccine vector. MolTher. 2014;22(2):420-429. [PMC free article] [PubMed]

[14] Miyamoto S, Inoue H, Nakamura T, et al. Coxsackievirus B3 is an oncolytic virus with immunostimulatory properties that is active against lung adenocarcinoma. Cancer Res. 2012;72(10):2609-2621. [PubMed]

[15] Hata Y, Etoh T, Inomata M, Shiraishi N, Nishizono A, Kitano S. Efficacy of oncolytic reovirus against human breast cancer cells. Oncol Rep. 2008;19(6):1395-1398. [PubMed]

[16] Norman KL, Coffey MC, Hirasawa K, et al. Reovirusoncolysis of human breast cancer. Hum Gene Ther. 2002;13(5):641652. [PubMed]

[17] Yang WQ, Senger DL, Lun XQ, et al. Reovirus as an experimental therapeutic for brain and leptomeningeal metastases from breast cancer. Gene Ther. 2004;11(21):1579-1589. [PubMed]

[18] Pan D, Marcato P, Ahn DG, et al. Activation of p53 by chemotherapeutic agents enhances reovirusoncolysis. PLoS One. 2013;8(1):e54006. [PMC free article] [PubMed]

[19] Pan D, Pan LZ, Hill R, et al. Stabilisation of p53 enhances reovirus-induced apoptosis and virus spread through p53-dependent NFkappaB activation. Br J Cancer. 2011;105(7):1012-1022. [PMC free article][PubMed]

[20] Wilcox ME, Yang W, Senger D, et al. Reovirus as an oncolytic agent against experimental human malignant gliomas. J Natl Cancer Inst. 2001;93(12):903-912. [PubMed]

[21] Roulstone V, Twigger K, Zaidi S, et al. Synergistic cytotoxicity of oncolytic reovirus in combination with cisplatin-paclitaxel doublet chemotherapy. Gene Ther. 2013;20(5):521-528. [PMC free article][PubMed]

[22] Pandha HS, Heinemann L, Simpson GR, et al. Synergistic effects of oncolytic reovirus and cisplatin chemotherapy in murine malignant melanoma. Clin Cancer Res. 2009;15(19):6158-6166. [PubMed]

[23] Heinemann L, Simpson GR, Boxall A, et al. Synergistic effects of oncolytic reovirus and docetaxel chemotherapy in prostate cancer. BMC Cancer. 2011;11:221. [PMC free article] [PubMed]

[24] 21. Kilani RT, Tamimi Y, Hanel EG, et al. Selective reovirus killing of bladder cancer in a co-culture spheroid model. Virus Res. 2003;93(1):1-12. [PubMed]

[25] Sei S, Mussio JK, Yang QE, et al. Synergistic antitumor activity of oncolytic reovirus and chemotherapeutic agents in non-small cell lung cancer cells. Mol Cancer. 2009;8:47. [PMC free article][PubMed]

[26] Hirasawa K, Nishikawa SG, Norman KL, Alain T, Kossakowska A, Lee PW. Oncolytic reovirus against ovarian and colon cancer. Cancer Res. 2002;62(6):1696-1701. [PubMed]

[27] Alain T, Hirasawa K, Pon KJ, et al. Reovirus therapy of lymphoid malignancies. Blood. 2002;100(12):4146-4153. [PubMed]

[28] Marcato P, Shmulevitz M, Lee PW. Connecting reovirusoncolysis and rassignaling. Cell Cycle. 2005;4(4):556-559. [PubMed]

[29] Shmulevitz M, Marcato P, Lee PW. Unshackling the links between reovirusoncolysis, rassignaling, translational control and cancer. Oncogene. 2005;24(52):7720-7728. [PubMed]

[30] Shmulevitz M, Pan LZ, Garant K, Pan D, Lee PW. Oncogenic ras promotes reovirus spread by suppressing IFN-beta production through negative regulation of RIG-I signaling. Cancer Res. 2010;70(12):4912-4921. [PubMed]

[31] Shmulevitz M, Marcato P, Lee PW. Activated rassignaling significantly enhances reovirus replication and spread. Cancer Gene Ther. 2010;17(1):69-70. [PubMed]

[32] Marcato P, Shmulevitz M, Lee PW. Connecting reovirusoncolysis and rassignaling. Cell Cycle. 2005;4(4):556-559. [PubMed]

[33] Marcato P, Shmulevitz M, Pan D, Stoltz D, Lee PW. Ras transformation mediates reovirusoncolysis by enhancing virus uncoating, particle infectivity, and apoptosis-dependent release. MolTher. 2007;15(8):1522-1530. [PubMed]

[34] Gujar SA, Marcato P, Pan D, Lee PW. Reovirus virotherapy overrides tumor antigen presentation evasion and promotes protective antitumor immunity. Mol Cancer Ther. 2010;9(11):2924-2933. [PubMed]

[35] Prestwich RJ, Ilett EJ, Errington F, et al. Immune-mediated antitumor activity of reovirus is required for therapy and is independent of direct viral oncolysis and replication. Clin Cancer Res. 2009;15(13):4374-4381. [PMC free article] [PubMed]

[36] Prestwich RJ, Errington F, Ilett EJ, et al. Tumor infection by oncolytic reovirus primes adaptive antitumor immunity. Clin Cancer Res. 2008;14(22):7358-7366. [PMC free article] [PubMed]

[37] Gujar SA, Pan DA, Marcato P, Garant KA, Lee PW. Oncolytic virus-initiated protective immunity against prostate cancer. MolTher. 2011;19(4):797-804. [PMC free article] [PubMed] 
[38] Gujar S, Dielschneider R, Clements D, et al. Multifaceted therapeutic targeting of ovarian peritoneal carcinomatosis through virusinduced immunomodulation. MolTher. 2013;21(2):338-347.[PMC free article] [PubMed]

[39] Errington F, Steele L, Prestwich R, et al. Reovirus activates human dendritic cells to promote innate antitumor immunity. J Immunol. 2008;180(9):6018-6026. [PubMed]

[40] Errington F, White CL, Twigger KR, et al. Inflammatory tumour cell killing by oncolytic reovirus for the treatment of melanoma. Gene Ther. 2008;15(18):1257-1270. [PMC free article] [PubMed]

[41] Hashiro G, Loh PC, Yau JT. The preferential cytotoxicity of reovirus for certain transformed cell lines. Arch Virol. 1977;54(4):307-315. [PubMed]

[42] Duncan MR, Stanish SM, Cox DC. Differential sensitivity of normal and transformed human cells to reovirus infection. J Virol. 1978;28(2):444-449. [PMC free article] [PubMed]

[43] Marcato P, Dean CA, Giacomantonio CA, Lee PW. Oncolytic reovirus effectively targets breast cancer stem cells. MolTher. 2009;17(6):972-979. [PMC free article] [PubMed]

[44] Hirasawa K, Nishikawa SG, Norman KL, et al. Systemic reovirus therapy of metastatic cancer in immune-competent mice. Cancer Res. 2003;63(2):348-353. [PubMed]

[45] wigger K, Vidal L, White CL, et al. Enhanced in vitro and in vivo cytotoxicity of combined reovirus and radiotherapy. Clin Cancer Res. 2008;14(3):912-923. [PubMed]

[46] Pan D, Pan LZ, Hill R, et al. Stabilisation of p53 enhances reovirus-induced apoptosis and virus spread through p53-dependent NFkappaB activation. Br J Cancer. 2011;105(7):1012-1022. [PMC free article][PubMed]

[47] Oncolytics Biotech Reolysin. [Accessed March 30, 2014]. Available from:http://www.oncolyticsbiotech.com/reolysin/default.aspx.

[48] Thirukkumaran CM, Nodwell MJ, Hirasawa K, et al. Oncolytic viral therapy for prostate cancer: efficacy of reovirus as a biological therapeutic. Cancer Res. 2010;70(6):2435-2444. [PubMed]

[49] OncolyticsBiotech . Oncolytics Biotech Inc. enters into sponsorship agreement with NCIC CTG for randomized phase II study in prostate cancer [press release] Calgary: Oncolytics Biotech; Feb 21, 2012. [Accessed March 16, 2014]. Available from: http://www.oncolyticsbiotech.com/English/news/press-release-details/2012/Oncolytics-Biotech-Inc-Enters-into-SponsorshipAgreement-with-NCIC-CTG-for-Randomized-Phase-II-Study-in-Prostate-Cancer.

[50] NCIC Clinical Trials Group Reolysin combined with docetaxel and prednisone or docetaxel and prednisone alone in metastatic castration resistant prostate cancer. [Accessed March 16, 2014]. from: http://www.clinicaltrials.gov/ct2/show/NCT01619813?term=reolysin\&rank=5. NLM identifier: NCT01619813.

[51] Forsyth P, Roldan G, George D, et al. A phase I trial of intratumoral administration of reovirus in patients with histologically confirmed recurrent malignant gliomas. MolTher. 2008;16(3):627-632.[PubMed]

[52] Kicielinski KP, Chiocca EA, Yu JS, Gill GM, Coffey M, Markert JM. Phase I clinical trial of intratumoralreovirus infusion for the treatment of recurrent malignant gliomas in adults. MolTher. 2014;22(5):1056-1062. [PMC free article] [PubMed]

[53] OncolyticsBiotech. Oncolytics Biotech announces positive interim safety results from Reolysin phase I malignant glioma study [press release] Calgary: Oncolytics Biotech; Dec 23, 2002. [Accessed March 16, 2014]. Available from: http://www.oncolyticsbiotech.com/English/news/press-release-details/2002/Oncolytics-Biotech-Announces-Positive-InterimSafety-Results-from-REOLYSIN-Phase-I-Malignant-Glioma-Study.

[54] OncolyticsBiotech. Oncolytics Biotech Inc. announces positive results of US phase 2 clinical trial investigating Reolysin in combination with paclitaxel and carboplatin in head and neck cancers [press release] Calgary: Oncolytics Biotech; Nov 14, 2011. [Accessed March 16, 2014]. Available from:http://www.oncolyticsbiotech.com/English/news/press-release-details/2011/OncolyticsBiotech-Inc-Announces-Positive-Results-of-US-Phase-2-Clinical-Trial-Investigating-REOLYSIN-in-Combination-with-Paclitaxeland-Carboplatin-in-Head-and-Neck-Cancers.

[55] Oncolytics Biotech Phase 2 study of Reolysin in combination with paclitaxel and carboplatin in patients with head and neck $\begin{array}{llll}\text { carcinoma. [Accessed March 16, 2014]. } & \text { Available }\end{array}$ from:http://www.clinicaltrials.gov/ct2/show/NCT00753038?term=reolysin\&rank=13. NLM identifier: NCT00753038.

[56] OncolyticsBiotech. Oncolytics Biotech Inc. announces positive top-line data from REO 018 randomized study of Reolysin in head and neck cancers [press release] Calgary: Oncolytics Biotech; Nov 21, 2013. [Accessed March 16, 2014]. Available from:http://www.oncolyticsbiotech.com/English/news/press-release-details/2013/Oncolytics-Biotech-Inc-Announces-Positive-TopLine-Data-from-REO-018-Randomized-Study-of-REOLYSIN-in-Head-and-Neck-Cancers

[57] Oncolytics Biotech Efficacy study of Reolysin in combination with paclitaxel and carboplatin in platinum-refractory head and neck $\begin{array}{llll}\text { cancers. [Accessed March 16, 2014]. Available } & \end{array}$ from:http://www.clinicaltrials.gov/ct2/show/NCT01166542?term=reolysin\&rank=3. NLM identifier: NCT01166542.

[58] Phelps M, Cohn D, O’Malley D, et al. Reovirus replication in ovarian and peritoneal tumors after intravenous administration. 2010. [Accessed May 21, 2014]. Available from:http://oncolytics.s3.amazonaws.com/data/46/original.pdf.

[59] OncolyticsBiotech. Calgary: Oncolytics Biotech; Dec 1, 2010. [Accessed March 16, 2014]. Oncolytics Biotech Inc. announces start of enrollment in randomized phase II ovarian cancer study [press release] Available from: http://www.oncolyticsbiotech.com/English/news/press-release-details/2010/Oncolytics-Biotech-Inc-Announces-Start-ofEnrollment-in-Randomized-Phase-II-Ovarian-Cancer-Study.

[60] National Cancer Institute Paclitaxel with or without viral therapy in treating patients with recurrent or persistent ovarian epithelial, or primary peritoneal cancer. [Accessed March 16, 2014]. from:http://www.clinicaltrials.gov/ct2/show/record/NCT01199263?term=reolysin\&rank=19. NLM identifier: NCT01199263.

[61] Morris DG, Feng X, DiFrancesco LM, et al. REO-001: A phase I trial of percutaneous intralesional administration of reovirus type 3 Dearing (Reolysin) in patients with advanced solid tumors. Invest New Drugs. 2013; 31(3):696-706. [PubMed]

[62] Gollamudi R, Ghalib MH, Desai KK, et al. Intravenous administration of Reolysin, a live replication competent RNA virus is safe in patients with advanced solid tumors. Invest New Drugs. 2010; 28(5):641-649. [PMC free article] [PubMed]

[63] Vidal L, Pandha HS, Yap TA, et al. A phase I study of intravenous oncolytic reovirus type 3 Dearing in patients with advanced cancer. Clin Cancer Res. 2008;14(21):7127-7137. [PubMed]

[64] Galanis E, Markovic SN, Suman VJ, et al. Phase II trial of intravenous administration of Reolysin(R) (reovirus serotype-3-Dearing strain) in patients with metastatic melanoma. MolTher. 2012;20(10):1998-2003. [PMC free article] [PubMed]

[65] Mita A, Sankhala K, Sarantopoulos J, et al. A phase II study of intravenous (IV) wild-type reovirus (Reolysin) in the treatment of patients with bone and soft tissue sarcomas metastatic to the lung. J ClinOncol. 2009;27(Suppl 15):10524.

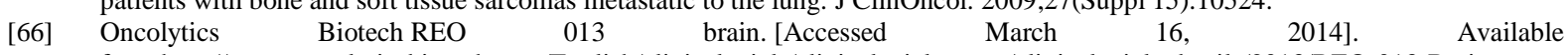
from:http://www.oncolyticsbiotech.com/English/clinical-trials/clinical-trials-news/clinical-trials-details/2013/REO-013-Brain. 
[67] OncolyticsBiotech . Oncolytics Biotech Inc. announces phase I multiple myeloma cancer study [press release] Calgary: Oncolytics Biotech; Mar 10, 2011. [Accessed March 16, 2014]. Available from:http://www.oncolyticsbiotech.com/English/news/press-releasedetails/2011/Oncolytics-Biotech-Inc-Announces-Phase-I-Multiple-Myeloma-Cancer-Study.

[68] National Cancer Institute Viral therapy in treating patients with relapsed or refractory multiple myeloma. [Accessed March 16, 2014]. Available from:http://www.clinicaltrials.gov/ct2/show/NCT01533194?term=reolysin\&rank=15. NLM identifier: NCT01533194.

[69] Karapanagiotou EM, Roulstone V, Twigger K, et al. Phase I/II trial of carboplatin and paclitaxel chemotherapy in combination with intravenous oncolytic reovirus in patients with advanced malignancies. Clin Cancer Res. 2012;18(7):2080-2089. [PubMed]

[70] White CL, Twigger KR, Vidal L, et al. Characterization of the adaptive and innate immune response to intravenous oncolytic reovirus (Dearing type 3) during a phase I clinical trial. Gene Ther. 2008; 15(12):911-920. [PubMed]

[71] Harrington KJ, Karapanagiotou EM, Roulstone V, et al. Two-stage phase I dose-escalation study of intratumoralreovirus type 3 Dearing and palliative radiotherapy in patients with advanced cancers. Clin Cancer Res. 2010; 16(11):3067-3077. [PMC free article] [PubMed]

[72] OncolyticsBiotech. Oncolytics Biotech Inc. announces positive results of UK phase II Reolysin and radiation combination clinical trial [press release] Calgary: Oncolytics Biotech; Apr 7, 2009. [Accessed March 16, 2014]. Available from: http://www.oncolyticsbiotech.com/English/news/press-release-details/2009/Oncolytics-Biotech-Inc-Announces-PositiveResults-of-UK-Phase-II-REOLYSIN-and-Radiation-Combination-Clinical-Trial.

[73] Lolkema MP, Arkenau HT, Harrington K, et al. A phase I study of the combination of intravenous reovirus type 3 Dearing and gemcitabine in patients with advanced cancer. Clin Cancer Res. 2011; 17(3):581-588. [PubMed]

[74] OncolyticsBiotech. Oncolytics Biotech Inc. completes patient enrollment in UK phase I clinical trial investigating Reolysin in combination with cyclophosphamide in patients with advanced malignances [press release] Calgary: Oncolytics Biotech; Aug 16, 2012. [Accessed March 16, 2014]. Available from:http://www.oncolyticsbiotech.com/news/press-release-details/2012/OncolyticsBiotech-Inc-Completes-Patient-Enrollment-in-UK-Phase-I-Clinical-Trial-Investigating-REOLYSIN-in-Combination-withCyclophosphamide-in-Patients-with-Advanced-Malignances/default.aspx.

[75] Monica M, Mita M, Wang Y, et al. A study of Reolysin in combination with gemcitabine in patients with advanced pancreatic adenocarcinoma. Mol Cancer Ther. 2011;10(Suppl):B55.

[76] OncolyticsBiotech. Oncolytics Biotech Inc. completes patient enrollment in US phase II clinical trial investigating Reolysin in combination with gemcitabine in patients with advanced or metastatic pancreatic cancer [press release] Calgary: Oncolytics Biotech; Oct 24, 2012. [Accessed March 16, 2014]. Available from: http://www.oncolyticsbiotech.com/English/news/press-releasedetails/2012/Oncolytics-Biotech-Inc-Completes-Patient-Enrollment-in-US-Phase-II-Clinical-Trial-Investigating-REOLYSIN-inCombination-with-Gemcitabine-in-Patients-with-Advanced-or-Metastatic-Pancreatic-Cancer.

[77] Oncolytics Biotech A study of Reolysin in combination with gemcitabine in patients with advanced pancreatic adenocarcinoma. [Accessed March 16, Available from:http://www.clinicaltrials.gov/ct2/show/NCT00998322?term=reolysin\&rank=10. NLM identifier: NCT00998322.

[78] Comins C, Spicer J, Protheroe A, et al. REO-10: A phase I study of intravenous reovirus and docetaxel in patients with advanced cancer. Clin Cancer Res. 2010;16(22):5564-5572. [PMC free article] [PubMed]

[79] Oncolytics Biotech A study of Reolysin in combination with paclitaxel and carboplatin in patients with squamous cell carcinoma of the lung. [Accessed March 16, Available from:http://www.clinicaltrials.gov/ct2/show/study/NCT00998192?term=reolysin\&rank=12. NLM identifier: NCT00998192.

[80] Oncolytics Biotech Study of Reolysin in combination with paclitaxel and carboplatin in patients with metastatic $\begin{array}{lllll}\text { melanoma. [Accessed } & \text { March } & 16, & 2014] & \text { Available }\end{array}$ from:http://www.clinicaltrials.gov/ct2/show/NCT00984464?term=reolysin\&rank=11. NLM identifier: NCT00984464.

[81] OncolyticsBiotech. Oncolytics Biotech Inc announces phase I study in pediatric patients with relapsed or refractory solid tumors to be conducted by the children's oncology group and sponsored by the national cancer institute [press release] Calgary: Oncolytics Biotech; Nov 18, 2010. [Accessed March 16, 2014]. Available from: http://www.oncolyticsbiotech.com/English/news/pressrelease-details/2010/Oncolytics-Biotech-Inc-Announces-Phase-I-Study-in-Pediatric-Patients-with-Relapsed-or-Refractory-SolidTumors-to-be-Conducted-by-the-Childrens-Oncology-Group-and-Sponsored-by-the-National-Cancer-Institute.

[82] National Cancer Institute Viral therapy in treating young patients with relapsed or refractory solid tumors [web site on the internet] US national institutes of health. [Accessed March 16, 2014]. Available from: http://www.clinicaltrials.gov/ct2/show/NCT01240538?term=reolysin\&rank=16. NLM identifier: NCT01240538.

[83] OncolyticsBiotech. Oncolytics Biotech Inc. announces positive Reolysin clinical trial data presented at ASCO gastrointestinal cancers symposium [press release] Calgary: Oncolytics Biotech; Jan 28, 2013. [Accessed March 16, 2014]. Available from: http://www.oncolyticsbiotech.com/English/news/press-release-details/2013/Oncolytics-Biotech-Inc-Announces-PositiveREOLYSIN-Clinical-Trial-Data-Presented-at-ASCO-Gastrointestinal-Cancers-Symposium.

[84] Oncolytics Biotech Study of Reolysin in combination with FOLFIRI and bevacizumab in FOLFIRI naive patients with KRAS mutant metastatic colorectal cancer. [Accessed March 16, 2014]. Available from: http://www.clinicaltrials.gov/ct2/show/NCT01274624?term=reolysin\&rank=1. NLM identifier: NCT01274624.

[85] OncolyticsBiotech. Oncolytics Biotech Inc. enters into sponsorship agreement with NCIC CTG for randomized phase II study in advanced and metastatic breast cancers [press release] Calgary: Oncolytics Biotech; Jun 21, 2012. [Accessed March 16, 2014]. Available from:http://www.oncolyticsbiotech.com/English/news/Press-Release-Details/2012/Oncolytics-Biotech-Inc-Enters-intoSponsorship-Agreement-with-NCIC-CTG-for-Randomized-Phase-II-Study-in-Advanced-and-Metastatic-Breast-Cancers.

[86] NCIC Clinical Trials Group A study of Reolysin for patients with advanced/metastatic breast cancer.[Accessed March 16, 2014]. Available from: http://www.clinicaltrials.gov/ct2/show/NCT01656538?term=reolysin\&rank=8. NLM identifier: NCT01656538.

[87] OncolyticsBiotech. Oncolytics Biotech Inc. enters into sponsorship agreement with NCIC CTG for randomized phase II study in colorectal cancer [press release] Calgary: Oncolytics Biotech; 2012. May 3, [Accessed March 16, 2014]. Available from: http://www.oncolyticsbiotech.com/English/news/press-release-details/2012/Oncolytics-Biotech-Inc-Enters-into-SponsorshipAgreement-with-NCIC-CTG-for-Randomized-Phase-II-Study-in-Colorectal-Cancer.

[88] NCIC Clinical Trials Group Reolysin in combination with FOLFOX6 and bevacizumab or FOLFOX6 and bevacizumab alone in $\begin{array}{lllll}\text { metastatic } & \text { colorectal } & \text { cancer. [Accessed } & \text { March 2014]. }\end{array}$ from:http://www.clinicaltrials.gov/ct2/show/NCT01622543?term=reolysin\&rank=9. NLM identifier: NCT01622543.

[89] OncolyticsBiotech. Oncolytics Biotech Inc. enters into sponsorship agreement with NCIC CTG for randomized phase II study in non-small cell lung cancer [press release] Calgary: Oncolytics Biotech; Jun 5, 2012. [Accessed March 16, 2014]. Available from: http://www.oncolyticsbiotech.com/English/news/press-release-details/2012/Oncolytics-Biotech-Inc-Enters-into-SponsorshipAgreement-with-NCIC-CTG-for-Randomized-Phase-II-Study-in-Non-Small-Cell-Lung-Cancer. 
[90] NCIC Clinical Trials Group Reolysin in patients with previously treated advanced or metastatic, non small cell lung cancer receiving standard salvage therapy. [Accessed March 16, 2014]. from:http://www.clinicaltrials.gov/ct2/show/NCT01708993?term=reolysin\&rank=4. NLM identifier: NCT01708993.

[91] Samuel CE. Reoviruses and the interferon system. Curr Top Microbiol Immunol. 1998; 233 (Pt 2):125-145. [PubMed]

[92] Kawai T, Akira S. Innate immune recognition of viral infection. Nat Immunol. 2006;7(2):131-137.[PubMed]

[93] Guidotti LG, Chisari FV. Noncytolytic control of viral infections by the innate and adaptive immune response. Annu Rev Immunol. 2001; 19:65-91. [PubMed]

[94] Morris G, Maes M. Mitochondrial dysfunctions in myalgic encephalomyelitis/chronic fatigue syndrome explained by activated immuno-inflammatory, oxidative and nitrosative stress pathways. Metab Brain Dis. 2014;29(1):19-36. [PubMed]

[95] Adair RA, Roulstone V, Scott KJ, et al. Cell carriage, delivery, and selective replication of an oncolytic virus in tumor in patients. Sci Transl Med. 2012;4(138):138ra77. [PMC free article] [PubMed]

[96] Fisher K. Striking out at disseminated metastases: the systemic delivery of oncolytic viruses. CurrOpinMolTher. 2006;8(4):301313. [PubMed]

[97] Ferguson MS, Lemoine NR, Wang Y. Systemic delivery of oncolytic viruses: hopes and hurdles. Adv Virol. 2012; 2012:805629. [PMC free article] [PubMed]

[98] Roy D, Bell J. Cell carriers for oncolytic viruses; current challenges and future directions. Oncolytic Virother. 2013; 2013(2):47-56.

[99] Jennings VA, Ilett EJ, Scott KJ, et al. Lymphokine-activated killer and dendritic cell carriage enhances oncolytic reovirus therapy for ovarian cancer by overcoming antibody neutralization in ascites. Int J Cancer. 2014; 134(5):1091-1101. [PMC free article] [PubMed]

IOSR Journal of Pharmacy and Biological Sciences (IOSR-JPBS) is UGC approved Journal with Sl. No. 5012, Journal no. 49063.

Bindhu Jayaprakash. "Evolution of Reovirus in Cancer Therapy." IOSR Journal of Pharmacy and Biological Sciences (IOSR-JPBS) 12.4 (2017): 6-14. 